\title{
Multiple Primary Tumors in Differentiated Thyroid Carcinoma and Relationship to Thyroid Cancer Outcome
}

\author{
ÖZGür ÖMÜR, ZEHRA ÖZCAN, BüLENT YAZICI, AYşEGüL AKGÜN, AYLIN ORAL AND HAYAL ÖZKILIÇ \\ Ege University Medical Faculty Department of Nuclear Medicine, İzmir, Turkey
}

\begin{abstract}
AIM: Patients with differentiated thyroid carcinoma (DTC) have long-life expectancy and are at risk for developing a second primary cancer. Aim of this study was to assess the occurrence of DTC in conjunction with other primary neoplasms. It was also aimed to explore the possibility of synchronous or metachronous other malignancies having an impact on clinical course of thyroid carcinoma. MATERIAL \& METHODS: Clinical records of 1680 DTC patients treated and followed in our institution over last twenty years were reviewed. Forty-five second primary tumors were found in 42 patients. These patients were classified into 3 groups as antecedent (group I), synchronous (group II) or subsequent (group III) according to the timing of occurrence of non-thyroidal malignancy. The initial characteristics of thyroid neoplasm were compared between patients with DTC plus another tumor and DTC only. Kaplan-Meier Survival Analysis was used to estimate the survival probability for patients with DTC alone and DTC plus another primary tumor. RESULTS: There were 15 synchronous and 30 metachronous tumors in 42 patients. Three of them had triple tumors. The most common second primary was lympho-haematological and upper aero digestive system tumors in group I and II respectively, whereas a variety of tumors were noted in group III. Despite the more common occurrence of unfavourable prognostic factors in patients with multiple cancers than thyroid cancer alone, complete response to radioiodine therapy and recurrence free survival rate was similar in both groups $(\mathrm{p}>0.05)$. CONCLUSION: The results of the current series imply that the occurrence of multiple primary tumors is not uncommon in patients with DTC. Close medical surveillance and the use of advanced screening modalities might lead to the detection of second primary tumors in DTC. However, the presence of second primary seems not to affect the clinical course of DTC.
\end{abstract}

Key words: Multiple primary tumors, Differentiated thyroid carcinoma, Clinical outcome

(Endocrine Journal 55: 365-372, 2008)

\begin{abstract}
AMONG all human carcinoma types, differentiated thyroid carcinoma (DTC) has a relatively benign biologic nature thus leading to long-life expectancy even in the presence of advanced disease. This unique characteristic may allow sufficient time for developing another cancer at another site in patients with DTC. While the use of radioiodine (RAI) or external radiotherapy for the treatment of DTC, in rare occasions, may offer the increased risk of second malignancy following treatment, the association of a variety of tumors occurring synchronously or metachronously also indicates the presence of different genetic and environ-
\end{abstract}

Received: September 16, 2007

Accepted: December 14, 2007

Correspondence to: Özgür ÖMÜR, Ege University Medical Faculty, Department of Nuclear Medicine, Bornova-İzmir, 35100 Turkey mental factors. At Memorial Sloan-Kettering Cancer Center, $50 \%$ of patients in whom an autopsy found a history of thyroid cancer, had a second and entirely separate cancer [1]. Recently, Ronckers et al. documented that association of thyroid carcinoma with a variety of primary extra-thyroidal cancers [2]. In their series derived from SEER cancer registries the authors showed positive associations between thyroid cancer and a variety of second primary cancers including breast, prostate, kidney, salivary glands, brain and CNS, scrotum and leukemia suggesting similar factors in terms of etiology and treatment effects [2].

In this study we aimed to review the occurrence of thyroid carcinoma in conjunction with other malignancies in a retrospective analysis of 1680 patients with DTC. Different cancers antecedent, concurrent or subsequent to the DTC were assessed in relation to the 
clinical presentation and the outcome. It was also aimed to explore the possibility that having a concurrent malignancy might affect clinical course of thyroid carcinoma when compared with patients having DTC alone.

\section{Material and Methods}

\section{Patients}

Clinical records of 1680 patients with DTC followed during the last 20 years (1986-2006) in our institution were reviewed. The mean follow-up was $7.4 \pm 6.2$ years and 550 of them had follow-up longer than ten years. Data regarding medical history, clinical presentation, results of diagnostic studies, therapeutic protocols and final clinical outcome were noted. As a part of our institutional protocol, radioiodine ablative therapy $75-100 \mathrm{mCi}(2.75-3.70 \mathrm{GBq})$ subsequent to near total thyroidectomy have been applied in patients with intra-thyroidal tumors exceeding $1.0-1.5 \mathrm{~cm}$. in diameter. Higher doses of radioiodine, in a range of 100-200 (3.7 to $7.4 \mathrm{GBq}$ ), have been used in persistent, recurrent disease or in case of distant metastases. RAI therapy has been repeated until ablation of all functioning thyroid remnant or metastatic tissues.

The retrospective evaluation of DTC patients revealed non-thyroidal primary malignancy in 42 of them. These patients were then divided into 3 groups according to the antecedent, synchronous or subsequent occurrence of the non-thyroidal tumor to DTC. Group I: Patients who had another primary malignancy diagnosed at least one year prior to the DTC. DTC was as a second primary neoplasm in this group of patients. Group II: Patients who had concurrent DTC and another primary malignancy diagnosed within the same year. Group III: Patients presenting with another non-thyroid malignancy at least one year after the DTC. Histological evaluation was obtained for all extra-thyroidal primary malignancies in order to exclude the possibility of metastasis of thyroid carcinoma. In this group of patients possible relation of second malignancy subsequent to the therapeutic RAI for DTC was investigated in terms of cumulative dose, time of appearance, histologic type and the clinical outcome.
Analysis

Initial characteristics and clinical outcome were comparatively assessed in patients with DTC plus another primary tumor and DTC alone. For this assessment, patients who had final clinical visit at the end of 2006 and completed follow-up longer than 1 year (n: 1009) were included. The characteristics of these DTC alone patients were then compared with patients who had DTC plus another primary tumor using student $t$ test and chi-square tests. Kaplan-Meier Survival Analysis was used to estimate the survival probability for patients with DTC alone and DTC plus another primary tumor. Recurrence free survival of 62 matching (with regard to age, gender, tumor histology and duration of follow-up) DTC alone patients was compared with 31 surviving patients with multiple malignancies. Every patient with multiple tumors was matched with 2 patients with single tumor.

\section{Results}

The retrospective documentation of the cohort indicated 45 synchronous or metachronous primary neoplasm in 42 (24 females and 18 males) of 1680 patients $(2.5 \%)$. Three of the patients had triple primary tumors (pts no: 1, 11 and 34 ). The mean age was 46.2 and 47.0 respectively at the time of the occurrence of first and second primary tumor.

\section{Group I}

Twenty patients had a previous history of another malignancy preceeding to the diagnosis of thyroid carcinoma (Table 1). There were 8 male and 12 female patients. The mean age was 39.4 at the time of first malignancy. The mean interval between first malignancy and DTC was $7.7(2-18)$ years. The details of patients' characteristics are presented in Table 1. Haematological malignancies including lymphoma, leukaemia and myeloma were the most common $(n=7)$ first primaries. Interestingly 6 of them were male patients. For the female patients, the most common initial primary malignancy was breast carcinoma. The thyroid malignancy was papillary carcinoma in 17 , follicular carcinoma in 1 and Hurtle cell carcinoma in 1 patient. A patient in this group (pt no: 1) had both follicular and papillary carcinoma foci in different lobes of thyroid 
Table 1. Clinical characteristics of group I patients (Age; at diagnosis of the primary tumor, M: Male, F: Female, AWD: Alive with disease, NEOD: No evidence of disease, LN: Lymph Node, Interval: The interval (year) between first malignancy and DTC), CNS: Central Nervous System, NHL: Non Hodgkin Lymphoma, ALL: Acute Lymphoid Leukemia, AML: Acute Myeloid Leukemia, * indicates patients have triple site cancer including thyroid carcinoma, EX: Exitus).

\begin{tabular}{|c|c|c|c|c|c|c|c|c|c|}
\hline No & Sex & Age & $\begin{array}{l}\text { Primary } \\
\text { neoplasm }\end{array}$ & $\begin{array}{c}\text { History of neck } \\
\text { radiation } \\
\text { before DTC }\end{array}$ & $\begin{array}{l}\text { Present status } \\
\text { of primary } \\
\text { tumor }\end{array}$ & $\begin{array}{l}\text { Thyroid tumor } \\
\text { histology }\end{array}$ & $\begin{array}{c}\text { Metastasis } \\
\text { of DTC }\end{array}$ & $\begin{array}{c}\text { Present status } \\
\text { of thyroid } \\
\text { cancer }\end{array}$ & $\begin{array}{c}\text { Interval } \\
\text { (year) }\end{array}$ \\
\hline $1 *$ & $\mathrm{~F}$ & 72 & Breast + stomach & - & EX & Papillary + Follicular & - & EX & 4 \\
\hline 2 & $\mathrm{~F}$ & 40 & Breast & - & NEOD & Papillary & - & NEOD & 15 \\
\hline 3 & $\mathrm{~F}$ & 45 & Breast & - & NEOD & Papillary & - & NEOD & 10 \\
\hline 4 & $\mathrm{~F}$ & 42 & Breast & - & NEOD & Papillary & - & NEOD & 2 \\
\hline 5 & $\mathrm{~F}$ & 42 & CNS tumor & + & NEOD & Follicular & - & NEOD & 6 \\
\hline 6 & $\mathrm{~F}$ & 47 & CNS tumor & + & EX & Papillary & - & EX & 4 \\
\hline 7 & $\mathrm{~F}$ & 42 & CNS tumor & - & NEOD & Papillary & - & NEOD & 10 \\
\hline 8 & $\mathrm{~F}$ & 48 & Cervix $\mathrm{Ca}$ & - & NEOD & Papillary & - & NEOD & 5 \\
\hline 9 & $\mathrm{~F}$ & 52 & Rectum & - & NEOD & Papillary & - & NEOD & 2 \\
\hline 10 & $\mathrm{~F}$ & 39 & Myelodysplasia & - & AWD & Papillary & - & NEOD & 6 \\
\hline $11^{*}$ & $\mathrm{~F}$ & 31 & Synovial Sarc. & + & Recurrence & Papillary & $\mathrm{LN}$ & NEOD & 17 \\
\hline 12 & $\mathrm{~F}$ & 19 & Nasopharynx & + & NEOD & Papillary & LN & NEOD & 4 \\
\hline 13 & M & 57 & Myelodysplasia & - & AWD & Papillary & bone lung & AWD & 10 \\
\hline 14 & M & 10 & AML & + & NEOD & Papillary & LN & AWD & 10 \\
\hline 15 & M & 3 & ALL & + & NEOD & Papillary & - & NEOD & 15 \\
\hline 16 & M & 69 & NHL & + & AWD & Papillary & LN & AWD & 4 \\
\hline 17 & M & 52 & NHL & + & AWD & Papillary & - & NEOD & 3 \\
\hline 18 & M & 12 & Hodgkin L. & + & NEOD & Papillary & LN & NEOD & 4 \\
\hline 19 & M & 16 & Osteosarcoma & + & NEOD & Papillary & - & NEOD & 18 \\
\hline 20 & M & 50 & Rectum Ca & - & Recurrence & Hurtle cell & $\mathrm{LN}$ & Recurrence & 5 \\
\hline
\end{tabular}

gland. For the details of the thyroid malignancy in group 1 patients, it was noted that 6 cases had regional lymph node metastasis and one had metastatic lung and bone involvement.

Ten of 20 patients had a previous history of radiotherapy including thyroid bed because of other primary neoplasm. The mean interval between external radiotherapy and DTC was 8.8 years (range 3-18). Histological type of DTC was papillary thyroid carcinoma in 9 of 10 cases and regional lymph node metastases were detected in 5 of them (50\%). An extreme case (pt no: 11 ) in this group had a history of radiotherapy due to synovial sarcoma on right supraclavicular region. Seventeen years after radiotherapy, in which thyroid bed was also included, papillary thyroid carcinoma in right thyroid lobe was detected. Interestingly, 19 years after initial primary, and 2 years after papillary thyroid cancer, a third malignancy, a fibrosarcoma was developed on external radiotherapy field (Table 1,3).

\section{Group II}

Fifteen patients had concurrent DTC and another pri- mary malignancy diagnosed within an interval less than 12 months (range: 0-12 month) (Table 2). There were 7 female and 8 male patients. The mean age was 52.2 years. The thyroid malignancy was papillary carcinoma in 14 and poorly differentiated follicular carcinoma in 1 patient. It was noted that 2 circumstances led to discovery of these synchronous malignancies. 1 . In 3 cases (pt no: $24,28,29$ ) thyroid malignancy was incidentally discovered during the operation for head and neck cancers. Thyroid tumor was less than $1.5 \mathrm{~cm}$ diameter in all of them. 2. In 8 DTC patients, just after the thyroid surgery, and in 4 patients with a history of non-thyroid malignancy, diagnostic work-up including imaging and laboratory studies led to the incidental detection of synchronous tumors. In this group of patients, squomous cell carcinoma of upper aerodigestive system was the most common malignancy followed by lung carcinoma.

\section{Group III}

Nine patients with DTC developed a second primary tumor after a mean interval of 3.8 years (range $2-$ 
Table 2. Clinical characteristics, histological type of thyroid carcinoma and other synchronous tumor of group II patients (SCC: Small Cell Carcinoma, NSCC: Non Small Cell Carcinoma, M. Myeloma: Multiple Myeloma, M. Melanoma: Malign Melanoma, * indicates patients have triple site cancer including thyroid carcinoma).

\begin{tabular}{|c|c|c|c|c|c|c|c|}
\hline No & Sex & Age & Other Primary Tm & $\begin{array}{c}\text { Present status of } \\
\text { other primary tumor }\end{array}$ & $\begin{array}{l}\text { Thyroid tumor } \\
\text { histology }\end{array}$ & $\begin{array}{c}\text { Metastasis } \\
\text { of DTC }\end{array}$ & $\begin{array}{c}\text { Present status of } \\
\text { thyroid cancer }\end{array}$ \\
\hline 21 & $\mathrm{~F}$ & 56 & Lung Ca (SCC) & EX & Follicular & $\mathrm{LN}$ & EX \\
\hline 22 & $\mathrm{~F}$ & 53 & Lung $\mathrm{Ca}$ (NSCC) & AWD & Papillary & - & NEOD \\
\hline 23 & $\mathrm{~F}$ & 56 & Lung $\mathrm{Ca}(\mathrm{SCC})$ & AWD & Papillary & - & NEOD \\
\hline 24 & $\mathrm{~F}$ & 51 & Parathyroid $\mathrm{Ca}$ & NEOD & Papillary & - & NEOD \\
\hline 25 & $\mathrm{~F}$ & 65 & Squamous cell Ca (Oral cavity) & NEOD & Papillary & $\mathrm{LN}$ & NEOD \\
\hline 26 & $\mathrm{~F}$ & 27 & Synovial Sarcoma & Recurrence & Papillary & - & NEOD \\
\hline 27 & $\mathrm{~F}$ & 28 & Tongue $\mathrm{Ca}$ & $\mathrm{EX}$ & Papillary & - & EX \\
\hline 28 & M & 53 & Larynx $\mathrm{Ca}$ & NEOD & Papillary & $\mathrm{LN}$ & NEOD \\
\hline 29 & M & 60 & Larynx $\mathrm{Ca}$ & NEOD & Papillary & $\mathrm{LN}$ & NEOD \\
\hline 30 & M & 54 & NHL (Stomach) & EX & Papillary & - & EX \\
\hline 31 & M & 56 & NHL & EX & Papillary & - & EX \\
\hline 32 & M & 75 & M. Myeloma & EX & Papillary & - & EX \\
\hline 33 & M & 49 & Epidermoid Ca (lip) & NEOD & Papillary & - & NEOD \\
\hline $34 *$ & M & 58 & M. Melanom & NEOD & Papillary & - & NEOD \\
\hline 35 & M & 42 & Malign Thymoma & EX & Papillary & - & EX \\
\hline
\end{tabular}

Table 3. Clinical and diagnostic properties of group III patients (age; at diagnosis of second primary tumor, Interval: The interval (year) between diagnosis of DTC and second neoplasm, MFH: Malign Fibrous Hystiocytoma, CML: Chronic Myeloid Leukemia, ${ }^{*}$ indicates patients have triple site cancer including thyroid carcinoma).

\begin{tabular}{cccccccccc}
\hline No & Sex & Age & $\begin{array}{c}\text { Thyroid tumor } \\
\text { histology }\end{array}$ & $\begin{array}{c}\text { Metastasis } \\
\text { of DTC }\end{array}$ & $\begin{array}{c}\text { RAI dose } \\
(\mathrm{mCi})\end{array}$ & $\begin{array}{c}\text { Present status of } \\
\text { thyroid cancer }\end{array}$ & Second tm & $\begin{array}{c}\text { Present status of } \\
\text { second tumor }\end{array}$ & $\begin{array}{c}\text { Interval } \\
\text { (year) }\end{array}$ \\
\hline 11 & F & 50 & Papillary & LN & 175 & NEOD & Fibrosarcoma & AWD & 2 \\
$34^{*}$ & M & 60 & Papillary & - & 100 & NEOD & Kidney tm. & NEOD & 2 \\
36 & F & 54 & Papillary & - & - & NEOD & Endometrium & NEOD & 3 \\
37 & F & 44 & Hurtle cell & - & 100 & EX & Breast Ca & EX & 3 \\
38 & F & 68 & Hurtle cell & - & 100 & NEOD & M. Melanom & NEOD & 3 \\
39 & F & 64 & Papillary & - & 100 & NEOD & Colon Ca & AWD & 9 \\
40 & F & 42 & Papillary & - & 100 & NEOD & CML & AWD & 4 \\
41 & M & 49 & Papillary & LN & 575 & EX & MFH & EX & 7 \\
42 & M & 74 & Follicular & - & 100 & EX & M. Myeloma & EX & 2 \\
\hline
\end{tabular}

9 years) (Table 3 ). Six of them were female and 3 were male. Eight of them had received RAI therapy after thyroid surgery. However, also in a patient with papillary microcarcinoma who did not receive RAI therapy and only followed with TSH suppression following surgery (patient no: 36) a second primary tumor was developed 3 years after thyroid tumor detection. The details of the group III patients are presented in Table 3. The thyroid malignancy was papillary carcinoma in 6 , follicular carcinoma in 1 and hurtle cell carcinoma in 2 patients. None of the patients in this group had received external radiotherapy in addition to RAI therapy. There was only one patient with a considerably higher cumulative RAI dose $575 \mathrm{mCi}(21.3 \mathrm{GBq})$ due to DTC with multiple lymphatic involvements in mediastinum. While a favourable clinical course of thyroid malignancy was noted in all these patients, non-thyroid malignancy subsequent to the DTC resulted in aggressive course in 3 patients and finally in the fatal outcome.

An extreme case in this group who is still alive had a history of renal transplantation (pt no: 34). Twelve years after renal transplantation, malign melanoma and papillary thyroid carcinoma were detected synchronously. Two years after these synchronous tumors, a third malignancy, renal cell carcinoma of the native kidney was developed. 
Presenting Characteristics and Outcome of Thyroid Carcinoma in Patients with and without Other Primary Neoplasms

The characteristics of thyroid carcinoma patients with and without other primary tumors are presented in Table 4 . While the age of presentation was nearly similar in both groups, male/female ratio was significantly higher in patients who had DTC plus another tumor. The most common thyroid cancer histology was papillary carcinoma in both groups. On the other hand, hurtle cell thyroid carcinoma was significantly more frequent in subjects with DTC plus another tumor ( $p=0.0012$, chi square test). Unfavourable factors including lymph node involvement and invasion into surrounding soft tissues was also significantly more common in patients with multiple tumors than DTC alone $(p=0,0024$ and 0,01 respectively, chi square test). Although the difference is short of statistical significance, distant metastasis of thyroid carcinoma was more frequent in cases with DTC alone ( $p=0.072$, chi square test).

When the clinical course of the disease was comparatively evaluated in both groups, it was noted that the complete remission rate was same in patients with and without other primary neoplasm despite the unfavourable features of DTC $(p=1.32$, chi square test). The frequency of recurrence was relatively higher, but sta-

Table 4. Characteristics of thyroid cancers in patients with thyroid cancer alone and cases with other synchronous or metachronous malignancies (* indicates statistical significance by student $t$ test and chi-square test)

\begin{tabular}{lcc}
\hline & $\begin{array}{c}\text { DTC with other } \\
\text { neoplasm }\end{array}$ & DTC alone \\
\hline Number of patients & 42 & 1009 \\
Female (\%)* & 57.1 & 83.7 \\
Male (\%) & 42.9 & 16.3 \\
Age at diagnosis of DTC & $49.2 \pm 14.5$ & $45 \pm 19.8$ \\
Thyroid carcinoma histology & & \\
Papillary (\%) & 83.3 & 85.6 \\
Follicular (\%) & 7.2 & 9.7 \\
Hurtle cell (\%)* & 7.2 & 3.3 \\
Other (\%) & 2.3 & 1.4 \\
Invasion into soft tissue (\%)* & 16.6 & 9.3 \\
LN metastasis (\%)* & 26.2 & 11.3 \\
Distant metastasis (\%) & 2.4 & 4.3 \\
Treatment Characteristics & & \\
Complete Remission (\%) & 87.1 & 90.3 \\
Recurrence (\%) & 3.2 & 2.6 \\
\hline
\end{tabular}

tistically insignificant, in patients with DTC plus another tumor than patients with DTC alone $(p=0.96$, chi square test). This was verified by comparing recurrence free survival of two groups matched with age, gender and histological type of DTC using KaplanMeier survey analysis. The characteristics of matching patients with DTC alone and DTC plus another tumor that were selected for survey analysis are presented in table 5. Recurrence free survival was not statistically different in these groups (Fig. 1).

\section{Discussion}

In this study the occurrence of different cancers antecedent, concurrent or subsequent to the DTC were reviewed in series of a single center over the last twentyyear period. Fifteen synchronous and 30 metachronous malignancies were noted in 42 of 1680 patients with differentiated thyroid carcinoma $(2.5 \%)$. Twenty patients had primary neoplasms before diagnosing their thyroid carcinoma. Ten of them had a history of external radiotherapy in the neck region. Although based on small number of patient, this indicates the increased risk for DTC in patients who were exposed to head and neck radiotherapy in their young ages. Moreover, in agreement with these observations mostly observed primary neoplasms in group I patients were lymphoma and leukemia followed by breast and CNS neoplasms. As previously noted, the radiation treatment related risks of thyroid cancer can be more commonly seen among survivors of leukemia and lymphoma [3]. On

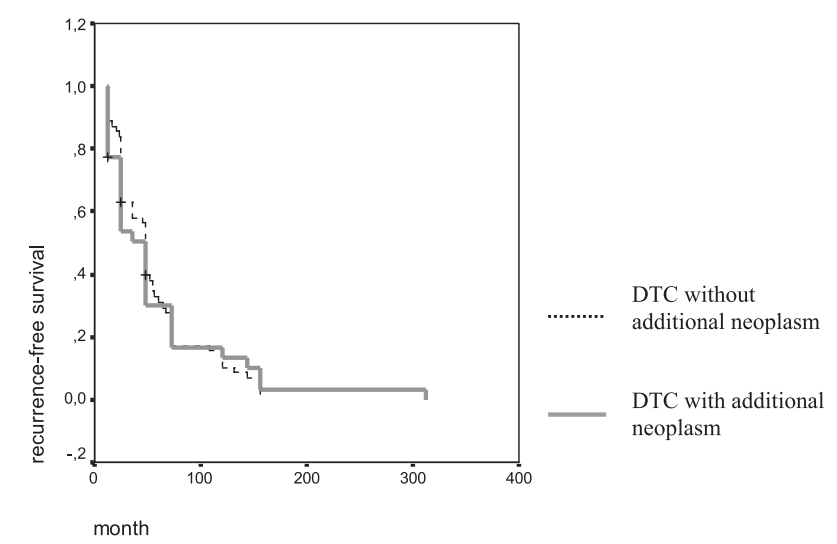

Fig. 1. Recurrence-free survival by Kaplan-Meier analysis in patients with DTC alone and DTC with additional tumors. Recurrence free survival was not statistically different in two groups. 
the other hand, even in lymphoma survivors not treated with external radiotherapy a significant increased risk of developing thyroid cancer has been defined possibly due to immune dysfunction or related factors [3]. In our patients, tumor histology was usually papillary carcinoma in previously irradiated patients and regional lymph node involvement was more frequent in agreement with previous reports $[4,5]$. Among the female patients of group I, breast carcinoma was the most common initial malignancy. Several studies have also suggested the possible association between thyroid and breast carcinoma in younger ages indicating the genetic, hormonal, environmental factors. In addition, therapy-related risks or some additional risks caused by close monitoring of these patients should be considered [6-9]. Higher risk for developing a second breast carcinoma after DTC or developing DTC after breast carcinoma was also documented suggesting the interrelationship of both tumors [6]. Recently, Park et al. documented higher incidence of thyroid cancer in patients with breast cancer than in the group who did not have breast cancer [10]. Therefore, the authors suggested the use of routine concurrent sonographic breast and thyroid examinations for detecting small thyroid tumors in both patient groups [10]. However, the effect of radiation delivered for the treatment of each of these neoplasms might increase the risk of subsequent thyroid or breast carcinoma [11].

In the present study, thyroid carcinoma and other non-thyroidal neoplasms were diagnosed synchronously in 15 patients (group 2). Six of them were initially presented with head and neck tumors. In 3 cases operated for head and neck cancer, papillary carcinoma of thyroid was detected per-operatively. Moreover, in 2 of them elective lymphadenectomy led to the discovery of isolated lymph node metastases of the thyroid tumor. Tumor size was less than $1.5 \mathrm{~cm}$ diameter in these cases. This observation indicates the importance of examining incidental thyroid pathologies in patients undergoing head and neck surgery [12]. In the remaining patients with head and neck tumors, thyroid lesions were detected during the detailed physical examination and imaging studies performed just prior to or after surgery. This is in agreement with previous reports emphasizing the importance of routine preoperative evaluation of the thyroid gland before open partial laryngectomy, especially with ultrasonography, to screen for occult synchronous thyroid lesions in patients with laryngeal tumors $[13,14]$.
Table 5. Details of patients' characteristics of 62 matching DTC alone and 31 surviving patients with multiple malignancies who were analyzed with Kaplan-Meier Survival Analysis.

\begin{tabular}{lccc}
\hline & $\begin{array}{c}\text { DTC with } \\
\text { other } \\
\text { neoplasm }\end{array}$ & $\begin{array}{c}\text { DTC } \\
\text { alone }\end{array}$ & $\mathrm{p}$ \\
\hline Number of patients & 31 & 62 & \\
Female (\%) & 64.5 & 62.6 & $>0.05$ \\
Male (\%) & 35.5 & 37.4 & $>0.05$ \\
Age at diagnosis of DTC & $49.4 \pm 14.8$ & $48.2 \pm 16.8$ & $>0.05$ \\
Thyroid carcinoma histology & 93.5 & 92.4 & $>0.05$ \\
Papillary (\%) & 0 & 2.4 & $>0.05$ \\
Follicular (\%) & 6.5 & 5.2 & $>0.05$ \\
Hurtle cell (\%)* & 0 & 0 & $>0.05$ \\
Other (\%) & \multicolumn{3}{c}{ Chi Square test } \\
& \multicolumn{3}{c}{} \\
\hline
\end{tabular}

In the majority of patients, second primary tumors were incidentally discovered during the sectional imaging studies performed for the assessment of extent of the disease. This implies the possibility of detecting a second primary tumor while evaluating the presence of local or distant metastatic involvement of a malignant disease which is not uncommon due to the use of advanced screening modalities studies $[15,16]$. In group II patients, lung carcinoma was the most second concurrent malignancy. In all cases chest $\mathrm{CT}$ evaluation led to the discovery of incidental nodules in lungs and lymph node enlargement which were verified histologically. FDG PET imaging with its ability to detect most malignancies in any part of the body led to the detection of an incidental thyroid tumor in a malignant melanoma patient (pt no: 34) in this series. Recently Nishiyama et al. also have documented the importance of FDG PET imaging for the detection of simultaneous second tumors and suggested that its use may change the patient management in head and neck cancers [16]. This also emphasizes that the increased medical surveillance may have significantly contributed to the detection of second primary tumors that would not otherwise have led to the clinically apparent disease.

According to database of the three major European cohorts, the risk of solid tumors and leukemia was enhanced in patients with DTC who received I-131 therapy [17]. Previous studies reported an increased risk of second cancers of the genitourinary tract [18-20], endocrine glands, nervous system [19], digestive tract, bone and soft tissue [17], salivary gland [17, 20] and adrenal gland [20] in patients treated radioiodine for 
DTC. In the current study, second primary tumors were developed in 9 patients 2-9 years after thyroid carcinoma. All of them had received therapeutic radioiodine except one patient with papillary microcarcinoma.

While the therapeutic use of radiation or radioiodine might be attributed to the development of secondary tumors, the casual relationship is unlikely in this study. Because, the radiation related secondary cancers usually appear after 10 years. However, in the majority of our cases the interval between radioiodine and secondary tumor was less than 5 years. Therefore we suggest that the occurrence of second primary tumors following DTC might not reflect the influence of radioiodine treatment. This was probably due to frequent medical surveillance since the tumors were detected in early stages of the disease. The cumulative radiation dose was also considerably less than the limits that may induce secondary tumors. Moreover, the occurrence of a second malignancy is noted even in a patient who has not received radioiodine. Recently Berthe et al. also documented lack of an association between radioiodine exposure and risk of second cancer after DTC [8]. The authors suggested that patient age and medical history of primary cancer as the significant risk factors for development of second cancers following DTC. However in another European study, a dose effect relationship between radioiodine exposure and the risk of second cancer was noted in colorectal cancer [21]. In our series, despite the low radioiodine dose, colon carcinoma was developed 9 years after in only one patient with DTC. The other second primary neoplasms were fibrosarcoma, malign fibrous histiocytoma, malign melanoma, renal cell carcinoma, multiple myeloma, breast, colon carcinoma and chronic myelocytic leukemia in agreement with the previous results in the literature $[17,18,20]$. The most dramatic case in this series (pt no: 11) had a previous history of external radiotherapy due to fibrosarcoma of supraclavicular region at the age of 31. Seventeen years after external radiotherapy which was covering right thyroid lobe, she developed first a thyroid tumor and 2 years later secondary sarcoma possibly due to external radiotherapy. Although could not be documented genetically, increased individual susceptibility to radiation might be a contributing factor in this case. However, despite the double second primaries induced by external radiotherapy she is still alive with disease. In the overall data, we noted less number of patients developing second cancer fol- lowing DTC compared to previous large series (99/ 2510 [20], 283/2968 [19], 576/6841 [21]) which may reflect the longer follow-up in these of series. Since a relatively shorter follow-up may result in underestimation of second primary tumors following DTC, we also examined the 550 DTC patients who had extended follow-up over 10 years in our institution. None of them developed a second primary tumor following RAI treatment as well.

The second goal of our study was to investigate the differences in the initial characteristics such as histological type, local or distant metastasis and clinical behavior of thyroid tumors in patients with DTC alone and DTC plus another primary tumor. Predominance of female over male was significantly lower in patients with DTC plus another primary tumor. However, age at diagnosis of thyroid cancer was the same in both groups. Histological type of the tumor was not significantly different except more common occurrence of hurtle cell histological subtype in patients with multiple primary tumors. Unfavorable prognostic factors at initial presentation such as regional lymph node involvement and invasion into surrounding soft tissues was significantly more frequent in patients with DTC plus other tumors. Despite these high risk prognostic factors $[22,23]$, the rate of complete response to radioiodine therapy and clinical outcome was not significantly different between two groups. Clinical behavior of thyroid tumor described as recurrent disease was also similar. Comparing recurrence free survival of all patients using Kaplan- Meier analysis also showed no significant difference (Fig. 1). This observation was also supported in a series reported by Mihailescu et al. [4]. While common occurrence of extrathyroidal invasion is noted in patients with DTC plus another tumor which may expect to increase local recurrence rate, this was not supported by statistical study. This observation may be related to the relatively shorter follow-up of these multiple tumor patients than those with DTC alone. On the other hand, the presence of multiple neoplasms might also lead to frequent medical examination and therefore early diagnosis and therapy in these patients.

In conclusion, second primary tumors accompanying DTC is not uncommon. This indicates the need for awareness of the possibility of developing additional cancers in this population. The close medical surveillance and the use of advanced imaging modalities might significantly contribute to the detection of sec- 
ond primary tumors. However, the clinical course of DTC seems not to be affected by the presence of additional primary tumors.

\section{References}

1. Strong E (1976) Discussion. Ann Surg 184: 553.

2. Ronckers CM, McCarron P, Ron E (2005) Thyroid cancer and multiple primary tumors in the SEER cancer registries. Int J Cancer 117: 281-288.

3. Jazbec J, Ecimovic P, Jereb B (2004) Second neoplasms after treatment of childhood cancer in Slovenia. Pediatr Bool Cancer 42: 574-581.

4. Mihailescu D, Shore-Freedman E, Mukani S, Lubin J, Ron E, Schneider AB (2002) Multiple neoplasms in an irradiated cohort: pattern of occurrence and relationship to thyroid cancer outcome. J Clin Endocrinol Metab 87: 3236-3241.

5. Tronko MD, Bogdanova TI, Komissarenko IV, Ebstein OV, Oliynyk V, Kovalenko A, Lkhtarev IA, Kairo I, Peters SB, Livolsi VA (1999) Thyroid carcinoma in children and adolescents in Ukraine after the Chernobyl nuclear accident-statistical data and clinicomorphologic characteristics. Cancer 86: 149-156.

6. Sadetzki S, Calderon-Margalit R, Peretz C, Novikov I, Barchana M, Papa MZ (2003) Second primary breast and thyroid cancers. Israel Cancer Causes Control 14: 367-375.

7. Turken O, Narin Y, Demirbaş S, Önde ME, Sayan O, Kandemir EG, et al. (2003) Breast cancer in association with thyroid disorders. Breast Cancer Res 5: 110113.

8. Smyth PP (2003) The thyroid, iodine and breast cancer. Breast Cancer Res 5: 235-238.

9. Volk N, Pompe-Kirn V (1997) Second primary cancers in breast cancer in Slovenia. Cancer Causes and Control 8: 764-770.

10. Park JS, Oh KK, Kim EK, Chang HS, Hong SW (2006) Sonographic screening for thyroid cancer in females undergoing breast sonography. AJR Am J Roentgenol 186: 1025-1028.

11. Adjadj E, Rubino C, Shamsaldim A, Le MG, Schlumberger M, de Vathaire F (2003) The risk of multiple primary breast and thyroid carcinomas. Cancer 98: 1309-1317.

12. Resta L, Piscitelli D, Fiore MG, Di Nicola V, Fiorella ML, Atavilla A, Marzullo A (2004) Incidental metastases of well-differentiated thyroid carcinoma in lymph nodes of patients with squamous cell head and neck cancer: eight cases with a review of the literature. Eur Arch Otorhinolaryngol 261: 473-478.

13. Farrag TY, Lin FR, Cummings CW, Sciubba JJ, Koch WM, Flint PW, Tufano RP (2006) Importance of routine evaluation of the thyroid gland prior to open partial laryngectomy. Arch Otolaryngol Head Neck Surg 132: 1047-1051.

14. Leon X, Sancho FJ, Garcia J, Sanudo JR, Orus C, Quer $M$ (2005) Incidence and significance of clinically unsuspected thyroid tissue in lymph nodes found during neck dissection in head and neck carcinoma patients. Laryngoscope 115: 470-474.

15. Houghton DJ, Hughes ML, Garvey C, Beasley NJ, Hamilton JW, Gerlinger I, Jones AS (1998) Role of chest CT scanning in the management of patients presenting with head and neck cancer. Head Neck 20: 614-618.

16. Nishiyama Y, Yamamoto $\mathrm{Y}$, Yokoe K, Miyabe K, Oqawa T, Toyama Y, Satoh K, Ohkawa M (2005) FDG PET as a procedure for detecting simultaneous tumors in head and neck cancer patients. Nucl Med Commun 26: 239-244.

17. Rubino C, de Vathaire F, Dottorini ME, Hall P, Schvartz C, Couette JE, Dondon MG, Abbas MT, Langlois C, Schlumberger M (2003) Second primary malignancies in thyroid cancer patients. $\mathrm{Br} \mathrm{J}$ Cancer 89: 1638-1644.

18. Berthe E, Henry-Amar M, Michels JJ, Rame JP, Berthet P, Babin E, Icard P, Samama G, Galateau-Salle F, Mahoudeau J, Bardet S (2004) Risk of second primary cancer following differentiated thyroid cancer. Eur J Nucl Med Mol Imaging 31: 685-691.

19. Hall P, Holm LE, Lundell G (1990) Second primary tumors following thyroid cancer. A Swedish recordlinkage study. Acta Oncol 29: 869-873.

20. Hall P, Holm LE, Lundell G, Bjelkengren G, Larsson LG, Lindberg S, Tennvall J, Wicklund H, Boice JD Jr (1991) Cancer risks in thyroid cancer patients. $\mathrm{Br} J$ Cancer 64: 159-163.

21. de Vathaire F, Schlumberger M, Delisle MJ, Francese C, Challeton C, de la Genardiere E, Meunier F, Parmentier C, Hill C, Sancho-Garnier H (1997) Leukaemias and cancers following iodine-131 administration for thyroid cancer. $B r J$ Cancer 75 : 734-739.

22. Cooper DS, Doherty GM, Haugen BR, Kloos RT, Lee SL, Mandel SJ, Mazzaferri EL, McIver B, Sherman SI, Tuttle RM (2006) The American Thyroid Association Guidelines Taskforce. Management Guidelines for Patients with Thyroid Nodules and Differentiated Thyroid Cancer. Thyroid 16: 109-142.

23. Shaha A (2006) Treatment of thyroid cancer based on risk groups. J Surg Oncol 94: 683-691. 